\title{
Massenkommunikation als Mittel christlicher Verkündigung
}

\author{
von Ulrich Saxer
}

\begin{abstract}
Aktualität, Information und Massenkommunikationsmittel bilden nach einer verbreiteten Ansicht einen modernen Zusammenhang und Ewigkeit, Verkündigung und Kirche einen traditionellen. Das Neben-, Gegen- oder Miteinander dieser beiden Komplexe empfinden indes seit einiger Zeit die aktiven Träger des christlichen Gedankengutes als immer dringlicheres Problem. ${ }^{1}$ Das Ewige zu aktualisieren, Verkündigung als Information auszugestalten und die kirchlichen Möglichkeiten der Telekommunikationsmittel viel intensiver $\mathrm{zu}$ nutzen, sind Hauptpostulate dieses neuen Medienaktivismus. Freilich unterschätzt dieser meist die Komplexheit der Massenkommunikationsphänomene und auch die Eigenmacht dieser vermeintlich unbedenklichen Mittel. Worauf sich die christliche Verkündigung tatsächlich einläßt, wenn sie die Institutionen der Massenkommunikation zu Hilfe ruft, soll deshalb im folgenden unter kultursoziologischen und publizistikwissenschaftlichen Gesichtspunkten etwas genauer untersucht werden.2
\end{abstract}

Dr. Ulrich Saxer ist Mitarbeiter der "Neuen Zürcher Zeitung ${ }^{*}$ und freier wissenschaft-. licher Mitarbeiter des Journalistischen Seminars der Universität Zürich. Für diese Zeitschrift schrieb er u. a. den Aufsatz über Messianismus und Wissenschaft bei Marshall McLuhan (CS $1: 1968$, S. 81-93). 


\section{Massenkommunikation als Mittel christlicher Verkündigung?}

[1. 1.] Nur dank Kommunikation, den distanzüberbrüdkenden Prozessen der Bedeutungsvermittlung, ist Gesellschaft möglich, und nicht minder Religion. Religionsgemeinschaften zumal sind Kommunikationsgemeinschaften in auffälligem Maß: Kommunikation mit dem Göttlichen ist, zumindest theoretisch, ihre raison d'être und entsprechende Kommunikation, Verkündigung', unter sich und nach außen ihre Haupttätigkeit.

Kommunikation, zwischenmenschliche, setzt aber ihrerseits umgekehrt auch wieder Gesellschaft voraus. Vielerlei Gemeinsamkeiten - darauf weist schon die Wortwurzel "communis" - müssen gegeben sein, damit der mit Kommunikation gemeinte Bedeutungsaustausch zustande kommt. Neben den technischen Voraussetzungen wie räumlicher Nähe oder einer Vermittlungsapparatur bedarf es dazu vor allem eines mentalen Mit- und Zueinanders der etwaigen Partner, das entsprechende Tatbestände und Situationen nahelegen. Für die religiöse Gruppe oder Gesellschaft hieße dies, daß sie nicht, wie sie vielleicht selbst glauben möchte, ausschließlich in Kommunikation gründet, sondern daß $\mathrm{zu}$ ihrer Konstituierung sehr viele andere sozio-kulturelle Faktoren beitragen müssen.

Aus diesem Interdependenzverhältnis resultiert, daß Gesellschafts-, Gruppen- und Individualverfassungen als Kommunikationsphänomene und -produkte und daß Kommunikationsverfassungen als Dimension von Gesellschaften, Gruppen und Individuen auszulegen sind. Die gesellschaftliche Machtverteilung beispielsweise wird sich also auch im Kommunikationswesen niederschlagen, Kommunikationsprivilegierung andererseits Machtpositionen stützen. Solche Kommunikationsprivilegien beinhalten ja nicht nur den Besitz besserer Informationen, die ein wirkungsvolleres Handeln gestatten, sondern auch sozialpsychologischen Sondereinfluß dank erheblicherer Definitions-, Aktualisierungs- und Anweisungsmöglichkeiten. Wer nämlich die Chance hat, häufiger als andere über alle oder gar ausschließlich über bestimmte Materien autonom als Kommunikator Kommunikationen einzuleiten, genießt damit ja das Vorrecht, aus der Unendlichkeit möglicher Themen bestimmte dringlich zu machen, eben zu aktualisieren, und kann damit auch in besonderem Ausmaß die Tatbestände auf die ihm genehmen Kategorien festlegen und mit offenen oder versteckten Verhaltensanweisungen versehen, was normalerweise natürlich umgekehrt auch wieder seine sonstige Machtposition verstärkt. Die Phänomene Ideologie und Ideologisierung gründen offenbar zu einem guten Teil in diesen und weiteren Eigentümlichkeiten des Sachverhaltes Kommunikation.

Kirchliche Kommunikationsprivilegien, und gar an Massenkommunikationsmitteln, $\mathrm{zu}$ verlangen, den damit unweigerlich verbundenen Machtaspekt dabei aber $\mathrm{zu}$ übersehen, verrät andererseits Blindheit bezüglich der elementaren Sozialzusammenhänge von Kommunikation. Kommunikationsprivilegien $\mathrm{zu}$ erhalten, und $\mathrm{zwar}$ trotz einer schwachen eigenen Machtposition, wie viele Kirchenvertreter meinen, muß dagegen bedeuten, daß diese letztlich unter Auflagen oder für Gegenleistungen von den realen Gesellschaftsmächten zediert werden. ${ }^{4}$

Die dauernde Kommunikationsbedürftigkeit von Individuen, Gruppen und Gesellschaften wiederum erzwingt die Institutionalisierung von Kommunikation, während das zur Kommunikationsverfassung verfestigte Insgesamt von entsprechenden Institutionen auf diese Bedürfnisse zurïckwirkt. Der christliche Missionsauftrag erscheint da letztlich als entsprechende Aufforderung, weil nur durch institutionalisierte Kommunikation vorhandene religiöse Bedürfnisse wirksam abgesättigt und sinnver- 
wandte neue geweckt werden können. Freilich verfällt damit eben auch die Verkündigung jener bekannten und zumal von protestantischer Seite so heftig empfundenen Dialektik von Glaubensinnerlichkeit und institutioneller Verhärtung ${ }^{5}$, von Gottesreich und Jetztwelt.

Der Modus von Kommunikation, das Veröffentlichen, ist es offenbar, der dieses Interdependenzgeflecht recht eigentlich begründet. Er zeitigt nämlich jene gruppenhaft oder gesamtgesellschaftlich relevanten Bedeutungswelten, die in einem sehr viel weiteren als bloß einem politischen Sinn "Offentlichkeiten", partikuläre und gesamtgesellschaftliche, sind. Sie kennzeichnen und normieren als Universa vereinbarter Symbole Beschaffenheit, Sinn und Wert der Gegebenheiten, die für die Individuen, Gruppen und Gesellschaften von Belang sind. Dementsprechend ist natürlich das Mitgestaltungsrecht der einzelnen und Gruppen an diesen über Sein und Sollen der Welt befindenden Symbolsystemen status-entscheidend. Der diesbezügliche Anspruch der religiösen Gruppen erweist sich da als sehr umfassend. Diese, legitimiert durch ihre Kommunikation mit dem Göttlichen, prägen ja viel bewußter und in viel größerer Zahl als andere Gruppen den Tatbeständen und Dingen Bedeutungen, eben religiöse, auf und suchen diese dann in die weitere Offentlichkeit zu schleusen. Je nach der gesamtgesellschaftlichen Position der religiösen Gruppen kann dieses Kommunikationsverhalten wie etwa im Mittelalter eine entsprechende Umgestaltung, „Verchristlichung“, der jeweiligen Gesamtöffentlichkeit zustandebringen oder aber es wirkt darin exzentrisch.

Der Modus des Veröffentlichens ist es schließlich auch, der Mitteilungsgeber und -empfänger, Kommunikator und Rezipient im Kommunikationsprozeß zirkelhaft aneinander schließt und nicht etwa durch eine Einbahnstraße verbindet. Jeder Mitteilende, auch der Massenkommunikator, ist ja schon durch seinen Einbezug in die erwähnte Öffentlichkeit vom Publikum abhängig und stellt sich normalerweise auch noch zusätzlich auf dessen jeweilige Besonderheiten ein. Dieses wiederum nimmt die ihm geltenden Mitteilungen nur selektiv und vornehmlich gruppentypisch auf, und hauptsächlich durch Gruppeneinfluß, wenn auch indirekt, wirkt es z. B. auch auf die Massenkommunikatoren zurück. ${ }^{6}$ Auch der Code, in dem die Mitteilungen abgefaßt sind, und das Medium, das etwaige technische Hilfsmittel, das sie transportiert, setzen zur erfolgreichen Verständigung Vereinbarungen und gemeinschaftliche Leistungen voraus. Angesichts all dieser Interdependenz- und Zirkelverhältnisse läßt sich offenbar jene schlichte Auffassung vom gesellschaftlich autonomen priesterlichen Kommunikator, der in selbstloser Zeugenschaft das Wort Gottes, wenn auch neuerdings mit technischen Hilfen, verbreitet, nicht mit der Wirklichkeit vereinbaren. ${ }^{7}$

[1. 2.] Auch die Massenmedien stehen als technische Apparate wie als sozio-kulturelle Institutionen zur Gesellschaft, in der sie tätig sind, in Interdependenz. Die Kommunikationsprozesse, die sie befördern, runden sich freilich nicht mehr sichtbarlich zu Kommunikationszirkeln, sondern nur noch mittelbar, d. h. über viele Stationen außerhalb des augenfälligen Kommunikationsprozesses im engern Sinn; dieser scheint vielmehr völlig einseitig vom Massenmedium aus auf passive atomisierte MassenPublica hin zu verlaufen. Eine gewisse Parallele zur klassischen, d. h. priesterlichen Verkïndigung in der Kirche ist evident, der Irrtum, die Massenmedien lediglich als eine Art von Verstärkern für die vorgegebene christliche Botschaft zu verstehen, schon von hier aus begreiflich. Lange Zeit und mehrheitlich auch heute noch war und ist denn auch dies die gängige Ansicht der die Massenkommunikationsmittel benützenden Prediger. 
Die immer umfänglichere Institutionalisierung von Massenkommunikation bedeutet indes, daß diese Massenverbreitungsmittel entwickelt wurden und eingesetzt werden, um den gesteigerten Kommunikationsbedarf der sich industrialisierenden oder schon industrialisierten Gesellschaften dauernd zu decken. Durch die institutionelle Verfassung der Massenmedien, sei sie liberal, demokratisch kontrolliert oder autoritär, wirkt die sozio-kulturelle Struktur dieser Gesellschaften. Immer notwendiger großbetrieblich organisiert, Objekte und Subjekte von Machtkämpfen, in die mannigfaltigsten Konkurrenzverhältnisse verstrickt, produzieren die Massenkommunikationsmittel scheinbar selbstherrlich ihr riesenhaftes Mitteilungsangebot, das aber in Wahrheit doch durch und durch von Gesellschaftsprozessen geprägt ist. Wenn es nun zudem zutrifft, daß diese industrielle Gesellschaft weitgehend säkularisiert, d. h. der christlichen Botschaft entfremdet ist, so müssen Massenkommunikation und Massenmedien offenbar auch säkulare Mechanismen sein. Der Rückzug der Kirche aus der industriellen Gesellschaft und der Siegeszug der Massenkommunikationsmittel in ihr fallen wohl nicht zufälligerweise in die gleiche Epoche. Konsequenterweise ist heute noch die in der christlichen Verkündigung gewöhnlich bekundete Haltung gegenüber den Massenmedien vorwiegend negativ. Ein Großteil der Medienproduktion, der unterhaltender Art ist, stößt auf seelsorgerliche Ablehnung.

Damit werden also die Massenkommunikationsmittel traditionellerweise von theologisch-kirchlicher Seite einesteils als willkommene Verkündigungsverstärker und andernteils als seelsorgerisches Ärgernis aufgefaßt. Die Folge davon ist das bekannte mehrdimensionale Bemühen katholischer und protestantischer Christen, Einfluß auf die Institutionsstruktur und Produktion der Massenmedien zu gewinnen, eine eigene christliche Produktion und die christlich-kirchliche Offentlichkeitsrepräsentation in ihnen zu gewährleisten und seelsorgerisch die Menschen gegen als schädlich eingeschätzte Medienproduktionen $\mathrm{zu}$ immunisieren. Dementsprechend beträchtlich ist in vielen Ländern auch das Aufgebot von Kirchenvertretern in den Aufsichts- und Programmgremien, hat der Kirchenfunk einige Prozent der Gesamtsendezeit zu relativ selbständiger Verfügung, sind an die Stelle kleiner Gemeindeblätter z. B. in Deutschland gewichtige regionale und überregionale christliche Zeitungen getreten, wird den Medienkonsumenten von dieser christlich-kirchlichen Publizistik überdies ständig Buch-, Film-, Radio- und Fernsehkritik angeboten, und all dies schließlich mit dem Erfolg, daß tatsächlich die Käufer- und Teilnehmerzahlen diejenigen der regelmäßigen Kirchgänger erheblich übersteigen.

Gemessen an den Hoffnungen der Verkündigenden und einer nominell zu 95 v. H. christlichen Gesellschaft ist dieser Erfolg allerdings nur relativ und vieldeutig dazu. Der unbestreitbare direkte und indirekte kirchliche Einfluß innerhalb der Medieninstitutionen und vornehmlich in zensurierender Art, auch deren Produktion kann ja ebensosehr auf dem Entgegenkommen der dominierenden Gesellschaftsmächte oder auf dem machtpolitischen Geschick der Vertreter der Institution und pressure group "Kirche" beruhen wie auf der Überzeugungskraft ihrer Botschaft und dem Bedarf der anvisierten Rezipienten. Genau wie bei der Großorganisation Kirche ist ja auch bei der institutionalisierten Massenkommunikation trotz diesbezüglicher Marktforschung die Rückkoppelung der wirklichen Publikumswünsche ins Bewußtsein der Kommunikatoren schwach. Darum muß auch die Frage meist offen bleiben, ob z. B. die teilweise beachtlichen Empfängerzahlen bei religiösen Sendungen tatsächlich von ungesättigten religiösen Bedürfnissen herrühren oder von der bekannten schichtspezifischen Gewohnheit, allen Medien und möglichst vielen Mitteilungstypen sich auszusetzen. ${ }^{8}$ Blosse Produktions- und Rezipientenzahlen sagen auf jeden Fall noch 
sehr wenig über das reale Resultat der massenverbreiteten Verkündigung aus, ihre häufige und optimistische Zitierung durch Kirchenvertreter dagegen um so mehr über das zähe Fortleben der alten Verstärkertheorie. ${ }^{9}$

[1.3.] Sicher sind ja die Massenmedien als technische Apparate und wenigstens dem Namen nach „Mittel“, aber sie wirken eben mit größter Intensität und unvermeidlich auf die Gesellschaft, die sich ihrer bedient, und auf die Ereignisse, die sie „vermitteln" sollen, auch wieder zurïck. So wie etwa die „Public Relations“ genannte Kommunikationsstrategie eigens „Ereignisse" fabriziert, um durch diese zusätzliche Offentlichkeitsrepräsentation in den Massenmedien zu erlisten, so ist bei der christlichen Beanspruchung derselben plötzlich auch das Was der christlichen Verkündigung problematisch geworden. An diesen vermeintlich mitteilungsindifferenten Verstärkern ist unversehens auch eine seither vielbeklagte inhaltliche Krise der christlichen Verkündigung deutlich geworden. ${ }^{10}$

Freilich ist auch die christlich-kirchliche Einsicht, die Massenmedien als Anstoß zum Neü̈berdenken des eigenen Verkündigungsauftrages und seiner Inhalte zu verstehen, noch ungenügend. Schon längst ist die Institution Kirche selbst für ihr Welt- und damit auch ihr Selbstverständnis in radikale Abhängigkeit von den Diensten der Massenmedien geraten. Wo aber diese unreflektiert einfach für Mittel einer sich autonom dünkenden christlichen Verkündigung gehalten werden, da ereignet sich leicht gerade das Umgekehrte, indem, ihr selbst nicht bewußt, die christliche Verkündigung zum Mittel der Manager der Massenkommunikation wird.

Chance und Gefahr, die der christlichen Religion aus ihrer Interaktion mit den Massenmedien erwachsen, sind also, daß sie dabei in ihrem zentralen Auftrag, dem kommunikativen, mit der modernen Welt in ihrer vielleicht repräsentativsten Gestalt sich auseinandersetzen kann, dies aber in einem falschen, d. h. teils ideologisierten, teils einfach wirklichkeitsfremden Bewußtsein tut. Die auf die Telekommunikationsmittel in doppelter Hinsicht angewiesene Kirche ist ihnen nämlich auch in doppelter Weise ausgeliefert. Unvermeidlich werden im Strom der von ihnen mitgeteilten Aktualitäten die Akzente anders gesetzt, als die Kirche zu ihrem Welt- und Selbstverständnis bedürfte, und umgekehrt begibt sich die Kirche, indem sie ihre Verkündigung den Massenmedien anvertraut, mit Notwendigkeit auch in deren Zweideutigkeit. Eitel wäre jedenfalls die Hoffnung, vorindustrielle, mithin strukturfremde Kultur-, insbesondere Religionsziele einfach über das moderne Mittel der Massenkommunikation in der neuzeitlichen Gesellschaft verwirklichen zu können.

\section{Kirchliche und industriegesellschaftliche Kommunikationsverfassung}

[2. 1.] In der Kommunikationsverfassung der industriellen Gesellschaft sind sowohl deren Institutionsgefüge als auch die sie dominierenden historischen Trends irgendwie wirksam. Dem muß die christliche Verkündigung, die als Teil dieser Kommunikationsverfassung agiert und zugleich auf diese reagiert, Rechnung tragen. Für das gesellschaftsbezogene christliche Kommunikationshandeln dürften dabei einesteils die Umfunktionalisierung und teilweise Entleerung des primären sozialen Horizontes wie Dorf und Familie durch die ständige Zunahme daseinserhaltender und -gestaltender Großorganisationen und andernteils die mit den Schlagworten „Entideologisierung“, „Fundamentaldemokratisierung" und „Konsumkultur" charakterisierbaren Trends von besonderer Bedeutung sein. Schließlich muß auch das Verkündigungs- 
hauptziel berüdksichtigt werden, das sich für den modernen Protestantismus ungefähr als Verpflichtung der Rezipienten auf ein selbst- und weltverantwortliches Dasein in stetem Transzendenzbezug umschreiben ließe, wozu im Katholizismus noch eine besondere Verantwortung gegenüber der Kirche käme.

Die traditionelle Verkündigung spricht sich mündlich oder über das lokale Blättchen aus: für den primären sozialen Horizont. Diese Kommunikationssituation ist im Grunde vorindustriell, aber dem personhaft-ganzheitlichen Mitteilungswillen gemäß. Solche Verkündigung verwandelt sich heute fast von selbst in Einzel- und Kleingruppenseelsorge.

Verkündigung durch Massenkommunikationsmittel zielt dagegen aus dem sekundären Horizont teils sich scharf konkurrenzierender, teils sich obligarisch stützender Großorganisationen in den dem Massenkommunikator weitgehend unbekannten Primärbereich, ist doch der Medienempfang in Demokratien ein kaum kontrolliertes Privatvergnügen. Die von vielen Kirchenvertretern ernst genommenen Metaphern von der Radio- oder Fernseh-,Gemeinde“ bzw. dem Radio- oder Fernseh-„Gespräch“ sind angesichts dieser prinzipiellen Veränderung der Kommunikationssituation durch das Massenmedium nicht ungefährliche Fiktionen, die den Verkündigungserfolg beschränken oder verfälschen können, weil sie wirklichkeitswidrig immer noch die unmittelbare $\mathrm{Z}$ weiseitigkeit der vorindustriellen Kommunkationssituation voraussetzen.

Freilich gibt es genügend Kniffe, diese gewaltige Distanz zwischen Massenkommunikatoren und Rezipienten zu überspielen, und daß die Medienfachleute, darunter auch kirchlich beauftragte, diese mit Erfolg und oft geringen Skrupeln einsetzen, dafür zeugen die in jeder Leser-, Hörer- oder Zuschauerpost bekundeten Vertrautheitsansprüche gegenüber dem Massenmedium. Die "Gemeinde", die etwa der allseits beliebte Hörfunk- oder Fernsehpfarrer so begründen kann, ist indes dem Fan-Club ähnlicher als der Dorfgemeinde. Die durch das Massenmedium als pseudo-persönliche Beziehung gestaltete Verkündigung ermuntert genau wie die politische und die kommerzielle Werbung das Publikum zur verharmlosenden Personalisierung des entfremdeten sekundären Horizonts. Daß die von den Massenmedien vermittelte Offentlichkeit wesentlich ein mit versteckten Direktiven gespicktes Produkt des Konkurrenzkampfes von Großorganisationen ist, wird dabei ebenso vertuscht wie sanktioniert. Die wechselseitigen Anleihen von Verkündigung und Werbung sind unter diesem Gesichtspunkt nicht mehr verwunderlich, sondern entsprechen bloß dem konsequenten Pragmatismus ferner geistlicher bzw. kommerzieller Eliten, welche glauben, nur durch Ausbeutung von dessen Illusionen ein Publikum ansprechen zu können. ${ }^{11}$

Gestehen die durch ein Massenmedium Verkündigenden dagegen sich und ihrem Publikum, daß sie als Sprechvertreter einer Institution (unter andern Institutionen) entfernte, anonyme Privatleute beeinflussen wollen, was die Nachbarschaft zu Werbespot und politischem Programm ja ohnehin verrät, dann wird die Kirche nicht um eine Klärung ihres eigenen institutionellen Selbstverständnisses und ihrer Beziehungen zu den andern Institutionen herumkommen, und zwar für sich wie für ihre Zuhörer. Weiterhin etwa bloß Privatwerten das Wort zu reden, nachdem man doch für sich ein Massenkommunikationsprivileg durchgesetzt hat, ist wenig glaubwürdig. In seiner Portion Sendezeit die andern Emissionen und die für sie verantwortlichen Organisationen vage, aber dauernd zu tadeln, in der Gewißheit, von diesen trotzdem weitgehend geschont zu werden, ist ein fragwürdiges Wettbewerbsverhalten. Gesellschaftskritik, schwach dokumentiert oder rücksichtsvoll allgemein, verpufft im Leeren und verschlechtert zugleich das sozialpsychologische Klima. Ganz anders als 
Parteien und Verbände sein $\mathrm{zu}$ wollen und doch mit ihnen am runden Tisch, im selben Programmheft, mit verwandten Methoden aufzutreten, ist verwirrend. „Information" zu nennen, was doch als Missionierung gedacht ist, läßt am publizistischen Ethos der Betreffenden zweifeln. Konsum-Askese zu predigen, wo die eigene TVoder Zeitungsverkündigung vielleicht durch eine entsprechende Werbung mitfinanziert wird, ist inkonsequent. Solche und weitere publizistische Fehlleistungen ${ }^{\mathbf{2}}$ bezeugen unmißverständlich das bekannte Institutionalisierungsdilemma der Kirche, das ihre Mitkonkurrenten in der Massenkommunikation nicht quält.

[2. 2.] Vor allen andern muß also die Kirche ihre eigene Kommunikationsverfassung bedenken, will sie sich in dem von ihr erhofften Sinn erfolgreich in die Massenkommunikation einschalten. Dazu gehört allerdings von Anfang an die Erkenntnis, daß - mutatis mutandis - in der kirchlichen Kommunikationsverfassung die erwähnten Distanzphänomene und Trends ähnlich zu Tage treten und sich auswirken wie in der gesamtgesellschaftlichen. Die gesamtgesellschaftliche Kommunikation verbessern zu wollen, wie unter dem Zauberwort „Gespräch“ sehr viele kirchliche Kreise sich bemühen, dürfte angesichts der Tatsache, daß die Kirche selbst wie nicht gleich eine zweite Institution an internen Kommunikationsschwierigkeiten leidet, nicht leicht fallen. Wenn andererseits der Geist mehr oder weniger weht, wie er will, dann hält an der Institution nur noch die durchaus weltliche Organisation, und diese ist es dann auch, die über den christlichen Massenmedieneinsatz in entsprechender Weise befindet.

Auch yon der sogenannten Entideologisierung ist die Kirche mitergriffen worden. Diese bedeutet nun nicht so sehr Versachlichung denn Pragmatisierung des Handelns unter Verzicht auf dessen systematische Rechtfertigung. Die äußerst rudimentäre fachtheologische Erhellung des Komplexes Massenkommunikation ${ }^{13}$ erinnert da fatal an die eingestandene Grundsatzlosigkeit der politischen Parteien. Andererseits entbehrt z. B. die unablässige Verketzerung „bloßer Worte“ zugunsten der offenbar unproblematischen Taten im Massenmedium und durch eine Institution, die sich vornehmlich als das Wort verkündigende versteht, nicht einer gewissen Paradoxie. Informationsethisch könnte sich eine Kirche, die ihrem Publikum zur Mündigkeit verhelfen will, vielleicht auch einmal fragen, ob etwa die gängigen publizistischen Objektivitätsregeln nicht gerade zur Ideologie werden, wenn die neuen Medien immer aggressivere Aktualisierungsmöglichkeiten gestatten, welche den durch Objektivität ermöglichten Distanzierungseffekt wieder vereiteln.

Die Fundamentaldemokratisierung schließlich problematisiert das Verhältnis des kirchlichen Massenkommunikators zur industriegesellschaftlichen Kommunikationsverfassung noch einmal in entscheidender Weise. Darunter ist die immer breitere Streuung von politischer Macht, Wirtschafts-- und Kulturgütern zu verstehen, die allerdings an der Struktur des industriellen Betriebs ihre harte Schranke findet. Sozialpsychologisch hat sich damit ein allgemeines Anspruchsklima herausgebildet, auf das die protestantische Kirche ihrer institutionellen Verfassung und ihrer Herkunft gemäß hauptsächlich mit ablehnendem Befremden, der klagenden Kulturkritik $^{\mathbf{1 4}}$ ähnlich, reagiert hat und teilweise noch reagiert.

Eine Sozialisierung der noch weitgehend auf das Pfarramt konzentrierten Autorität stößt beispielsweise auf große Schwierigkeiten. Der im Massenmedium tätige Geistliche wird denn auch, obwohl meist schon zu Unrecht, von Kollegen und Publikum vor allem in diesem Licht gesehen. Dabei verläßt er doch mit seinem Auftritt vor Mikrophon oder Kamera seinen kirchlichen Schutz- und Herrschafts- 
raum und reiht sich einfach unter die anderen Anbieter ein. De facto bestätigt er damit, auch noch im Konfessionsproporz der Sendezeiten, den demokratischen Weltanschauungspluralismus, den er dann freilich mit seinem of erhobenen Anspruch auf die letztlich einzige Wahrheit doch wieder dementiert. ${ }^{15}$

Konkurrenziert wird dabei durch die kirchliche Massenkommunikation unvermeidlich und in erster Linie der Gemeindepfarrer, dessen Predigtleistungen nun plötzlich neuen Vergleichsmaßstäben standhalten müssen. ${ }^{16}$ Kirchliche Massenkommunikation zersetzt damit möglicherweise das kirchliche Organisationsprinzip „Ortsgemeinde“, ist doch der Abbau des Provinzialismus eine der bedeutendsten Wirkungsrichtungen der Massenkommunikation.

\section{Christliche Verkündigung als Mittel der Massenkommunikation?}

[3. 1.] Auf Grund der allgemeinen Kennzeichnung der industriegesellschaftlichen und der kirchlichen Kommunikationsverfassung lassen sich noch einige spezifische Massenkommunikationsgegebenheiten anvisieren, welche die problematische Mittlerqualität der Massenmedien noch greller beleuchten und die Frage legitim erscheinen lassen, was delin nun eigentlich da wessen Mittel sei. Dies soll unter den Stichworten "Dialog", „Zeugenschaft", „Konversion" und „christliche Offentlichkeit" geschehen. Das Zirkelhafte, Dialogische jeder nicht bloß punktuellen Kommunikation erfüllt sich auch in der Massenkommunikation, aber nur mittelbar, nicht als "Gespräch". Den Selektionsakten seines potentiellen Publikums sucht jeder Massenkommunikator irgendwie $\mathrm{zu}$ entsprechen, er antizipiert, so gut es geht, dessen Reaktionen in der Gestaltung seiner Botschaft und kann überdies zusätzliche Aufschlüsse über dessen Eigenheiten durch Publikumsvertreter oder wissenschaftliche Publikumsanalysen erhalten. Die Klagen, die Kirche beantworte auch über das Massenmedium bloß Fragen, die niemand gestellt habe ${ }^{17}$, sind im Lichte der Teilnehmerzahlen übertrieben, aber als Zeichen einer besonders schwachen Rückkoppelung nicht unbedenklich. Gerade eine Institution wie die Kirche, die mit ihren Pfarrern gewissermaßen in jeder Gemeinde über „Korrespondenten" mit Sozialerfahrung verfügt, sollte imstande sein, diese Wissenssumme in Sendungen und Artikel einzuarbeiten, welche realen Bedürfnissen entsprechen.

Diese Tatsache hilft indes, im Unterschied zu den weltlichen Massenkommunikatoren, der Kirche nur bedingt, sollte doch ihre Verkündigung, wie schon deren durchaus eigentümlicher Rhythmus gemäß dem Kirchenjahr bezeugt, nicht bloß einfacher, sondern Doppeldialog, nämlich mit Gott und den Rezipienten, sein. Kommunikationstechnisch bedeutet dies eben unter anderm, daß statt des weltlichen Nachrichtenanfalls und Informationswertes der geistliche ihre publizistischen Beiträge bestimmt. Daraus resultiert zwangsläufig eine nur partiell bedürfnisorientierte und überdies ziemlich durchsichtige Selektionsstrategie im Bereich der referierwürdigen Geschehnisse, werden doch diese meist gemäß ihrem christlichen Demonstrationswert ausgewählt. Im Rahmen des modernen Informationsangebotes mutet dieses Verfahren unter Umständen tendenziös an.

Dazu kommt noch, daß der Verkündigende anders als alle anderen Massenkommunikatoren letztlich immer wieder ein und dieselbe Nachricht, die in der Bibel überlieferte, verbreiten muß, deren publizistischer Aktualitätswert vergleichsweise bescheiden ist. An entsprechenden Aktualisierungsversuchen der Hörfunk- und Fernsehpfarrer fehlt es nicht; das gewitzte Publikum indes wird sich durch diese Präsen- 
tationsweise nicht allzu of tödern lassen und überdies darin den profanen Gestus der kommerziellen Werbung, die das Immergleiche als das Niedagewesene ausgibt,

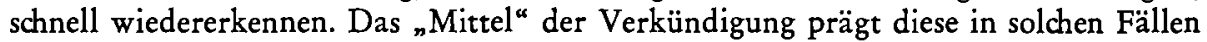
offenbar bis zur Unkenntlichkeit und Selbstverzerrung um, und der Diener am göttlichen Wort wird dabei zum Sklaven des menschlichen Aktualitätenmediums.

Die fast unerfüllbare publizistische Aufgabe des kirchlichen Massenkommunikators ist die Daueraktualisierung ein und desselben Themas in der Abstraktion und im Aktualitätenmilieu der Massenkommunikation und bei unklarer Bedürfnislage des Publikums. Während jedes Massenmedium im Grunde auf Neues aus ist, dieses freilich unter gestrigen Kategorien beleuchter, sieht die christliche Verkündigung, gerade umgekehrt, ein bestimmtes Stück Vergangenheit in immer wieder neuem Licht. Dies heißt zusätzlich, daß in der kirchlichen Publizistik der Kommentar den absoluten Vorrang vor der Information haben muß, was sie im Zeitalter der Entideologisierung in die Nähe des gegenwärtig rückläufigen Meinungsjournalismus und der verpönten Anweisungspublizistik bringt.

[3. 2.] Glaubwürdige Zeugenschaft wird unter diesen Umständen dem Verkündigenden wie seinem Massenmedienpublikum zum Problem. Uberhaupt läßt sich wohl die Verallgemeinerung wagen, daß die aus dem entfremdeten Raum der sekundären Systeme in den Primärbereich dringenden Massenmedien zur Veranschaulichung der Glaubenserfahrung oder gar zum Vorleben der Glaubensinnerlichkeit wenig geeignet seien. Der Kult erscheint schon durch seine Nachbarschaft zu Krimi und Sportschau nur noch als eine Möglichkeit unter andern; durch seine Ästhetisierung indes, wenn die Kamera geschmäcklerisch Kirchenfenster und andere Architekturreize heranholt und die Gläubigen kamerabewußt und dekorativ mit- und zugleich vorbeten, wird er für Mitwirkende und Zuschauende gar zur Show, zu einem weitern Stück Konsumkultur. ${ }^{18}$

Durch Anpassung ihrer Beiträge an die übrigen publizistischen Erfolgsmuster kann mithin die Kirche ihre Teilnehmerzahlen voraussichtlich steigern, aber eben um den Preis der Anpassung. Die gerade auch von Kirchenvertretern vielverketzerte "Sprache Kanaans" bezeugt da zwar sicher ständig eine beträchtliche Distanz zur Gegenwart, dafür bestätigt sie diese auch nicht einfach in ihrem So-Sein, wie es die häufigen und meist wenig kompetenten Anleihen beim sportlichen, militärischen oder politischen Jargon tun. Die verbreitete Auffassung, das Ankommen der christlichen Verkündigung sei im Grunde bloß eine „Verpackungsfrage“, ist bei Vertretern einer Religion, die auf dem Wort gründet, ziemlich befremdend und im Licht der Wirkungsforschung zweifellos ein Irrtum.18

Die entsprechende Medienarbeitsmaxime: "Je unkirchlicher, desto besser" kann denn auch nur als selbstkritische Einsicht in die kirchliche Distanz zur Industriegesellschaft, nicht aber zur Erreichung des kirchlichen Verkündigungszwecks nützlich sein. Wohl mag der eine oder andere Jugendliche durch den kirchlich offerierten Beat vor die Kanzel oder an die Verkündigungssendung gelockt werden. Entdeckt er aber, älter geworden, die also "verpackte" Absicht dahinter immer noch nicht, so wird er auch den gemeinten radikalen Unterschied dieser Sendung zur sonstigen Populärkultur, dem Fernsteuerungsversuch durch Konsumangebote, nie erfassen; durchschaut er dagegen den hinweisenden Sinn dieser christlichen Publizistik, wird er fortan verdrossen die Konsumverpackung der Anweisung, der er zu mißtrauen gelernt hat, auch mit der christlichen Verkündigung in eins setzen und sich wie schon seine politische nun auch seine christliche Abstinenz nicht mehr stören lassen. 
[3.3.] Alle diese mannigfaltigen christlichen Experimente mit den Massenmedien werfen unter immer neuen Aspekten die schon früher angesprochene Frage nach dem Selbst- und Weltverständnis der christlichen Verkündigung durch Massenkommunikation auf. Genügt es eigentlich der christlichen Massenkommunikation, einfach Publica zu gewinnen oder zielt sie wirklich auf deren Bekehrung zu Christus? Partizipation harmlos als Vorstufe zur Konversion zu interpretieren, ist in der Massenmedienstrategie nicht angängig, da sich die meisten Rezipienten von der Mehrzahl der Medienmitteilungen nur ihre vorgefaßten Meinungen und ohnehin schon eingenommenen Attitüden bestätigen lassen. ${ }^{20}$

Massen-Publica in den Medien zu gewinnen, wird auch der Kirche kaum mißlingen, wenn sie dafür nur unverzagt die bewährten Erfolgsmuster, am besten gleich diejenigen der Unterhalter, einsetzt. Diese verdanken freilich ihre breite Gefolgschaft den bescheidenen Ansprüchen, die sie stellen, und der konsequenten Aussparung des wirklich Strittigen. Die christliche Massenkommunikation, der Partizipation über alles geht, müßte sich also in Gestus und Gehalt dem in den Massenmedien so erfolgreichen System von Populärkultur ${ }^{21}$ einfügen und dient damit vielleicht sogar in gewisser Hinsicht dem sozialen Frieden. Selbst Kritik am Bestehenden kann sie dabei üben, aber eben immer nur im Rahmen jener Versöhnlichkeit, die letztlich ihr Einverständnis mit dem Status quo stets von neuem bezeugt. ${ }^{22}$

Reißt sie nämlich die vom beliebtesten Medienangebot gerade überdeckten kulturellen Abgründe und sozialen Konflikte rücksichtslos auf, so zerfällt sie nicht nur mit den (wie sie) die Massenmedien beliefernden mächtigen Organisationen, sondern auch mit einem Großteil ihres Publikums, das dann diese Programme ignoriert oder ablehnt.

Die Möglichkeiten, über die Mittel der Massenkommunikation auf eine Verchristlichung der Offentlichkeit hinzuwirken, sind also beschränkter und fragwürdiger, als der unter progressiven Kirchenvertretern gängige robuste Medienpragmatismus annimmt. Die Vorstellungen von der gewaltigen attitüdenverändernden Macht dieser Massenverbreitungsmittel, die ihm offenbar zugrundeliegen, verkennen den realen Wirkungsbereich eines demokratischen Massenmediensystems völlig. Bloß ein totalitäres kirchliches Medienmonopol brächte gegebenenfalls verhältnismäßig rasche und erhebliche Konversionswirkungen zustande. (Dennoch darf auch der Einfluß der demokratisch institutionalisierten Massenkommunikationsmittel nicht unterschätzt werden, nur ist er schwerer faßbar.)

Die demokratischen Massenmediensysteme vermitteln ja vor allem zwischen sekundärem und primärem Sozialhorizont, aber eben mit dem Gestus des Anbieters, nicht dem des Anweisers. An der von den Massenkommunikationsmitteln dominierten Offentlichkeit erleben daher deren Rezipienten stärker den Konsum denn die Direktive. Dies ist um so eher möglich, als diese Offentlichkeit als demokratische immerhin auf einem gewissen Konsens ruht. Als Teil des Systems sekundärer Großorganisationen sind die Massenmedien aber zugleich auch Mit-teiler der dort konzentrierten gesellschaftlichen Macht und funktionieren jedenfalls gewöhnlich weitgehend gemäß deren Wünschen, welche verständlicherweise gesamthaft auf die Erhaltung des Status quo zielen.

Der christlichen Verkündigung, die sich auf diese Mittel einläßt, droht die Gefahr der Komplizenschaft. Dann verabreicht sie einfach christliche Populärkultur und reduziert damit ihre Rezipienten noch einmal auf das, was sie ohnehin schon sind: Konsumenten. Kirchliche Weltannahme und kirchliche Weltbestätigung fallen damit in eins. Gestehen dagegen die Zeugen der christlichen Botschaft sich und ihrem 
potentiellen Publikum ihre und deren Distanz zur Welt der Gegenwart ein, so entfremden sie sich diesem Publikum wahrscheinlich noch stärker. In diesem Dilemma und manch weiterem müssen die Träger der christlichen Verkündigung den - oder einen - richtigen Weg finden. Das „Mittel“ prïft sie selbst.

\section{Anmerkungen:}

1. Die Fülle diesbezüglicher Bemühungen spiegelt sich z. B. in einer kaum mehr überblickbaren Vielzahl von Publikationen und Tagungen zu diesem Themenkomplex. Selbst die vielen Rezensionen und Berichte dazu, die in jeder Nummer von "Communicatio Socialis" erscheinen, vermögen dieses neue aktive Interesse an den Massenmedien nur teilweise zu erfassen. (Vgl. z. B. CS 1:1968, S. $6 \mathrm{ff} ., 277 \mathrm{ff} ., 359 \mathrm{f}$.; 2:1969, S. 44 f., 49.)

2. Dem Kommunikationswissenschaftler muß dabei freilich etwelches von der Soziologie und dem Nichttheologen etwelches von der Theologie nachgesehen werden. Die Massenkommunikationslehre hat ja trotz unzähliger empirischer Einzelstudien erst ein recht diffuses Bild der soziokulturellen Vorgänge um die Telekommunikationsmittel erbracht, weshalb sie auch von der Soziologie, wie z. B. René König, Grundlagenprobleme der soziologischen Forschungsmethoden, in: Sozialwissenschaft und Gesellschaftsgestaltung, Festschrift für Gerhard Weisser, Berlin 1963, S. 30 f., feststellt, nur zögernd integriert wird. Für die spezifisch religiöse oder christliche Massenkommunikation liegen die Verhältnisse gar besonders ungünstig, als hier vielen kirchlich-theologischen Aussagen, die indes fast immer spekulativ im Ansatz und normativ in der Ausrichtung sind, erst sehr wenige empirische Spezialuntersuchungen gegenüberstehen. Daran ist sowohl die Totalität der um Massenmedien und Religion zentrierten Phänomene als auch die u. a. von Helmut Schelsky, Religionssoziologie und Theologie, in: Auf der Suche nach Wirklichkeit, Düsseldorf-Köln 1965, S. 276 f., beschriebene radikale Verschiedenheit von Theologie und Soziologie schuld. Für eine Zusammenschau der verschiedenen Bereiche fehlen dementsprechend die integrationswissenschaftlichen Ansätze und Verfahren, weshalb es hier auch lediglich um die Formulierung einiger Thesen und nicht etwa um eine wirkliche Theorie gehen kann. Diese Thesen stellen ferner, als wirklichkeitswissenschaftliche, nicht den "richtigen", sondern den tatsächlichen und tatsächlich möglichen Einsatz der Massenmedien im Dienste christlicher Verkündigung zur Diskussion.

3. Unter "Verkündigung" soll hier im Unterschied $\mathrm{zu}$ theologisch gängigeren, fachspezifischeren Auslegungen das Insgesamt bewußt christlicher, d. h. an Gott und Christus orientierter und auf sie bezogener Kommunikationsbemühungen, und $\mathrm{zwar}$ vornehmlich von dazu irgendwie autorisierten (hier: protestantischen) Kommunikatoren, verstanden werden.

4. Dieses fragwürdige Verhältnis der Kirchen zum Machtaspekt von Kommunikation hat Helmut Thielicke, Leiden an der Kirche, Hamburg 1965, S. 185, auf die Formel gebracht: „Wir sind Pragmatisten der Einflußnahme...". Vgl. auch Walter Dirks, Das schmutzige Geschäft, Olten und Freiburg i. B. 1964, S. 24 und Eberhard Stammler, Der Journalist, in: Frömmigkeit in einer weltlichen Welt, hrsgg. v. Hans Jürgen Schultz, Stuttgart 1959, S. $274 \mathrm{f}$.

5. Vgl. z. B. die Beiträge von Th. F. O'Dea, Die fünf Dilemmas der Institutionalisierung der Religion, und von Friedrich Fürstenberg, Kirchenform und Gesellschaftsstruktur, in: Religionssoziologie, hrsgg. v. Friedrich Füstenberg, Neuwied/Berlin 1964.

6. Über die Massenkommunikationsprozesse und die darin wirksamen Interaktionen und Selektionsmechanismen orientieren im deutschen Sprachbereich unter verschiedenen Aspekten z. B. Film und Fernsehen im Spiegel der Wissenschiaft, hrsgg. v. Erich Feldmann und Ernst Meier, Gütersloh 1963; Friedhelm Neidhardt, Gesellschaftliche Wirkungen der Massenmedien, in: „Hamburger Jahrbuch für Wirtschafts- und Gesellschaftspolitik", 9. Jahr, Tübingen 1964, S. 210 f., Henk Prakke, Kommunikation der Gesellschaft, Münster 1968; Grundfragen der Kommunikationsforschung, hrsgg. .v. Wilbur Schramm, München 1964.

7. Dieser Irrtum prägt stärkstens das Konzildekret „Uber die Werkzeuge der sozialen Kommunikation", wie Otto B. Roegele in "Publizistik“, 9. Jg. (1964), H. 4, nachweist; aber auch Ambrosius K. Ruf, Fernsehen - Rundfunk - Christentum, Zürich 1960, und die in: Alte Botschaft - Neue Wege, hrsgg. v. Hans-Joachim Girock, Stuttgart 1966, schreibenden Autoren entrinnen ihm nicht immer. 
8. Theodore Peterson, Jay W. Jensen, William L. Rivers: The Mass Media and Modern Society, New York 1965, S. 129 f.

9. Vgl. z. B. Eberhard Stammler: Protestanten ohne Kirche, Hamburg 1967, S. 28.

10. Beiträge zu einer Rundfunkhomiletik, hrsgg. v. Manfred Josuttis, München 1967, allenthalben.

11. Vgl. Walter Dirks, a.a.O., S. 24 und Helmut Thielicke, a.a.O., S. 32 f.

12. Vgl. u. a. Eberhard Stammler: Der Journalist, a.a.O., S. 273-75; J. Daniel Hess: The Religious Journals' Image of the Mass Media, in: "Journalism Quaterly", Jowa City, Winter 1964; Roland E. Wolseley: Religion's Journalism in the USA, in "Gazette", Leiden, vol. XIII (1967), Nr. 3.

13. Manfred Josuttis, Vorwort des Herausgebers, S. 7; Claus Westermann, Rundfunkgemeinde und Kirchengemeinde, S. 53; Hans Jürgen Schultz, die unbewältigte Offentlichkeit der Kirche, S. 108; alle in: Beiträge zu einer Rundfunkhomiletik, a.a.O.

14. Kulturkritik übt, nach einem bekannten Wort von Arnold Gehlen („Studien zur Anthropologie und Soziologie", Neuwied a. R./Berlin 1963, S. 330) als Abwehrreflex eine "Schicht von Gebildeten, die in der technischen Gesellschaft in Gefahr gerät, sozial funktionslos zu werden, oder die ihre Rolle im gesellschaftlichen Ganzen nicht mehr definieren kann".

15. Friedrich Baumgärtel: Religion und Kirche im Rundfunk, in: Beiträge zu einer Rundfunkhomiletik, a.a.O., S. 26.

16. Kurt Frör: Die Predigt im Rundfunk, in: Beiträge zu einer Rundfunkhomiletik, a.a.O., S. 86.

17. Dorothee Sölle: Erwartung oder Enttäuschung - die Randsiedler, in: Alte Botschaft -Neue Wege, a.a.O., S. 35.

18. Hans Werner v. Meyenn: Bildpredigt oder Gottesdienst im Fernsehen, in „Rundfunk und Fernsehen", 3. Jg. (1955), 277 f.

19. Zu Sprachproblem und "Verpackungsideologie" vgl. z. B. Kirchensprache - Sprache der Kirche, hrsgg. v. Joachim Burckhardt, Zürich/Stuttgart 1964; Ulrich Gotthelf Fidk: Wort auf der Waage, Kleine Burckhardthaus-Bücherei, 1960 o. O.; Ulrich Oevermann: Schichtspezifische Formen des Sprachverhaltens und ihr Einfluß auf die kognitiven Prozesse, in: Begabung und Lernen, Deutscher Bildungsrat, Gutachten und Studien der Bildungskommission, Stuttgart 1969, S. 304 f.; Gerhardt Schnath: Modern um jeden Preis - die Jugendlichen, in: Alte Botschaft - Neue Wege, a.a. O., S. 57.

20. Vgl. z. B. Joseph T. Klapper: The Effects of Mass Communication, Glencoe 1960, allenthalben.

21. Zur Phänomenologie der Populärkultur vgl. z. B. Culture for the Millions, hrsgg. v. Norman Jacobs, Boston 21965 , und Mass Culture, hrsgg. v. Bernard Rosenberg und David M. White, Glencoe 1957.

22. Konrad Jutzler: Privatisierte Verkündigung, in: Beiträge zu einer Rundfunkhomiletik, a.a.O., S. 139.

\section{S U M M A R Y}

At the present time mass media by the Christian churches is looked upon far too readily as an auxiliary means for the proclamation of the faith. This new Christian pragmatism in the field of mass media very often is based on the wrong supposition that the telecommunications media simply intensifies the Christian message. But this media follows, like utterly secular institutions, their own regularities, who risk in different regards the integrity of the Christian message. It is impossible to create through them "community" in the common Christian sense, and it is further impossible for the Church to appear in a completely different field, like others who offer their ideas through mass media. There is the danger that mass media is not going to be in service for the proclamation of the faith but that the Christian faith is going to be a subject for mass media. 